\title{
Mid-regional pro-atrial natriuretic peptide and copeptin as indicators of disease severity and therapy response in CTEPH
}

\author{
Steffen D. Kriechbaum (10) ${ }^{1,2}$, Lillith Scherwitz ${ }^{1,2}$, Christoph B. Wiedenroth $^{3}$, \\ Felix Rudolph ${ }^{1,2}$, Jan-Sebastian Wolter ${ }^{1,2}$, Moritz Haas ${ }^{1,2}$, \\ Ulrich Fischer-Rasokat ${ }^{1,2}$, Andreas Rolf ${ }^{1,2,4}$, Christian W. Hamm ${ }^{1,2,4}$, \\ Eckhard Mayer ${ }^{3}$, Stefan Guth (10 ${ }^{3}$, Till Keller (10) ${ }^{1,2,4}$, Stavros V. Konstantinides (10) ${ }^{5,6}$, \\ Mareike Lankeit $\mathbb{1}^{5,7,8}$ and Christoph Liebetrau ${ }^{1,2,4,8}$
}

Affiliations: ${ }^{1}$ Kerckhoff Heart and Thorax Center, Dept of Cardiology, Bad Nauheim, Germany. ${ }^{2}$ German Center for Cardiovascular Research (DZHK), Partner Site Rhine-Main, Frankfurt am Main, Germany. ${ }^{3}$ Kerckhoff Heart and Thorax Center, Dept of Thoracic Surgery, Bad Nauheim, Germany. ${ }^{4} J u s t u s$ Liebig University Giessen, Medical Clinic I, Division of Cardiology, Giessen, Germany. ${ }^{5}$ Center for Thrombosis and Hemostasis, University Medical Center of the Johannes Gutenberg University, Mainz, Germany. ${ }^{6}$ Dept of Cardiology, Democritus University of Thrace, Alexandroupolis, Greece. ${ }^{7}$ Dept of Internal Medicine and Cardiology, Campus Virchow Klinikum (CVK), Charité - University Medicine Berlin, Berlin, Germany. ${ }^{8}$ These authors contributed equally.

Correspondence: Steffen D. Kriechbaum, Kerckhoff Heart and Thorax Center, Benekestr. 2-8, 61231 Bad Nauheim, Germany. E-mail: s.kriechbaumakerckhoff-klinik.de

\section{ABSTRACT}

Background: Chronic thromboembolic pulmonary hypertension (CTEPH) leads to right heart failure. Pulmonary endarterectomy (PEA) or balloon pulmonary angioplasty (BPA) restore pulmonary haemodynamics and allow cardiac recovery. This study examined the relationship of copeptin and midregional pro-atrial natriuretic peptide (MR-proANP) levels to disease severity and therapy response.

Methods: This observational cohort study included 125 patients (55 PEA/70 BPA) who underwent treatment and completed a 6-/12-month follow-up. Biomarkers, measured at baseline, prior to every BPA and at follow-up, were compared to 1 ) severe disease at baseline (right atrial pressure (RAP) $\geqslant 8 \mathrm{mmHg}$ and cardiac index $\leqslant 2.4 \mathrm{~L} \cdot \mathrm{min}^{-1} \cdot \mathrm{m}^{-2}$ ) and 2) optimal therapy response (no persistent pulmonary hypertension combined with a normalised RAP (mean PAP $\leqslant 25 \mathrm{mmHg}$, pulmonary vascular resistance (PVR) $\leqslant 3 \mathrm{WU}$ and RAP $\leqslant 6 \mathrm{mmHg}$ ) or a reduction in mean PAP $\geqslant 25 \%$, PVR $\geqslant 35 \%$ and RAP $\geqslant 25 \%$ ).

Results: Severely diseased patients had higher levels of MR-proANP (320 (246-527) pmol. $\mathrm{L}^{-1}$ versus 133 (82-215) $\left.\mathrm{pmol} \cdot \mathrm{L}^{-1} ; \mathrm{p}=0.001\right)$ and copeptin $\left(12.7(7.3-20.6) \mathrm{pmol} \cdot \mathrm{L}^{-1}\right.$ versus $\left.6.8(4.4-12.8) \mathrm{pmol} \cdot \mathrm{L}^{-1} ; \mathrm{p}=0.015\right)$ at baseline than the rest of the cohort. At baseline, MR-proANP (area under the curve (AUC) 0.91; cut-off value $227 \mathrm{pmol} \cdot \mathrm{L}^{-1}$; OR 56, 95\% CI 6.9-454.3) and copeptin (AUC 0.70; cut-off value $10.9 \mathrm{pmol} \cdot \mathrm{L}^{-1}$; OR 1.5, 95\% CI 1.2-1.9) identified severely diseased patients. After PEA/BPA, levels of MR-proANP (99 (58-145) $\left.\mathrm{pmol} \cdot \mathrm{L}^{-1} ; \mathrm{p}<0.001\right)$ and copeptin $\left(6.3(3.7-12.6) \mathrm{pmol} \cdot \mathrm{L}^{-1} ; \mathrm{p}=0.009\right)$ decreased and indicated optimal therapy response (MR-proANP $<123 \mathrm{pmol} \cdot \mathrm{L}^{-1}$ (AUC 0.70 ) and copeptin $<10.1 \mathrm{pmol} \cdot \mathrm{L}^{-1}$ (AUC 0.58)).

Conclusion: MR-proANP and copeptin levels are affected in CTEPH and decrease after therapy. MR-proANP identifies a severe disease status and optimal therapy response.

@ERSpublications

The assessment of cardiac stress and impact of therapy is crucial in CTEPH. Serum levels of MR-proANP are associated with haemodynamic disease severity and therapy response, and might thus support individualised patient management. https://bit.ly/2QKwb7x

Cite this article as: Kriechbaum SD, Scherwitz L, Wiedenroth CB, et al. Mid-regional pro-atrial natriuretic peptide and copeptin as indicators of disease severity and therapy response in CTEPH. ERJ Open Res 2020; 6: 00356-2020 [https://doi.org/10.1183/23120541.00356-2020].

Received: 5 June 2020 | Accepted after revision: 18 Aug 2020

Copyright $\odot$ ERS 2020. This article is open access and distributed under the terms of the Creative Commons Attribution Non-Commercial Licence 4.0. 


\section{Introduction}

Up to $4 \%$ of the patients who survive an acute pulmonary embolism are later diagnosed with chronic thromboembolic pulmonary hypertension (CTEPH) [1]. The persistence of pulmonary artery obstructions, compounded by pulmonary vascular remodelling, progressively impairs pulmonary haemodynamics [1-4]. Under these conditions, elevated pulmonary artery pressure (PAP) and pulmonary vascular resistance (PVR) burden the right heart and cause compensatory right heart remodelling [1-4]. Without treatment, this sequence leads to right heart failure, which is the major determinant of outcome in CTEPH [5]. It should be noted that CTEPH is the only potentially curable subtype of pulmonary arterial hypertension [1], and surgical pulmonary endarterectomy (PEA) is the treatment of choice [1]. In patients in whom surgery is not feasible, a sequence of medical therapy with riociguat and balloon pulmonary angioplasty (BPA) should be considered $[1,6]$. Both therapeutic approaches aim to restore pulmonary haemodynamics and thus allow right heart recovery to optimise patients' resilience and quality of life. A structured diagnostic work-up, including individualised risk stratification and assessment of therapy response, is challenging in daily clinical practice but crucial for an optimal outcome [1].

Noninvasive biomarkers of haemodynamic conditions and cardiac stress can provide valuable information in this context [7-10]. Atrial natriuretic peptide (ANP) is released upon cardiac (in particular, atrial) wall stress and has diuretic, vasodilatory and tissue-protective effects [11]. Further, an increase in circulating vasopressin is triggered by systemic stress, hypotension and an increase in plasma osmolality and impacts haemodynamics via peripheral vasoconstriction and renal reabsorption of sodium-free fluid [12]. Both biomarkers are characterised by low plasma protein stability, which limits the reproducibility of laboratory measurements and thus hampers their investigation in the context of cardiac diseases [12, 13]. Mid-regional pro-atrial natriuretic peptide (MR-proANP) and copeptin are by-products of ANP and vasopressin, respectively $[12,13]$. Their higher plasma stability allows a valid equimolar measurement as surrogates for the mature proteins. ANP and MR-proANP levels correlate with disease severity and outcome in heart failure [13], acute pulmonary embolism [14] and pulmonary hypertension [15-17]. Copeptin gained importance in the diagnostic work-up of acute cardiovascular disease, particularly acute coronary syndrome [18]. In addition, copeptin was identified as a strong predictor of outcome in acute pulmonary embolism $[19,20]$. Data on the relevance of MR-proANP and copeptin in patients with CTEPH are limited to animal models [21], small subcohorts and mixed pulmonary hypertension cohorts $[15,16,22,23]$. There are no data on the time course of changes in these biomarkers after CTEPH therapy. The aim of the current study was to investigate the levels of MR-proANP and copeptin in CTEPH patients treated with PEA or BPA and assess their association with disease severity and therapy response.

\section{Methods}

\section{Study population}

The present observational cohort study included 125 patients with confirmed CTEPH who were treated by BPA $(n=70)$ or PEA $(n=55)$ at the Kerckhoff Heart and Thorax Center, Bad Nauheim, Germany between 2014 and 2016 and who completed a 6-month follow-up (6-MFU) after the final BPA or a 12-month follow-up (12-MFU) after PEA. A total of 52 (74\%) patients of the BPA cohort and 16 (29\%) of the PEA cohort were also treated with riociguat. This was the result of the off-label character of other medication and the lack of any approved medication for CTEPH prior to 2014. Since then, riociguat has been approved for CTEPH treatment and recommended in the guidelines [1]. Thus, over time we adjusted our treatment approach and riociguat is administered for at least 3 months prior to possible BPA in inoperable CTEPH patients at our centre.

All patients were discussed in an interdisciplinary CTEPH conference to decide about the most appropriate individual treatment. The pre- and post-procedural management of the patients was recently published by our group $[8,24,25]$. All patients gave written informed consent. The ethics board of the Justus Liebig University of Giessen approved the study (AZ 43/14). The study protocol conforms to the ethical guidelines of the Declaration of Helsinki.

Pulmonary endarterectomy and balloon pulmonary angioplasty

PEA surgery and BPA interventional therapy were performed as standardised techniques. The detailed procedures have been published previously $\left(\mathrm{PEA}^{25} / \mathrm{BPA}^{24}\right)$.

\section{Right heart catheterisation}

Right heart catheterisation was routinely performed via the right internal jugular vein using a $6 \mathrm{~F}$ sheath and a standard Swan-Ganz catheter. The medication was not modified prior to or during the procedure. 


\section{Laboratory assessment}

Venous blood samples for biomarker analysis were collected in plain tubes at baseline prior to the PEA or first BPA procedure, before every consecutive BPA procedure in BPA patients, and at 6-MFU or 12-MFU. All measurements were carried out batch-wise on thawed samples by experienced staff blinded to patient characteristics.

Copeptin was measured in serum/plasma (EDTA, heparin) by TRACE (time-resolved amplified cryptate emission) technology (BRAHMS Copeptin proAVP KRYPTOR assay, Kryptor Compact Plus; BRAHMS $\mathrm{GmbH}$, Hennigsdorf, Germany): lower detection limit (LOD) $0.69 \mathrm{pmol} \cdot \mathrm{L}^{-1}$; standard curve range (SCR) 0.7-2000 $\mathrm{pmol} \cdot \mathrm{L}^{-1}$; intra-assay coefficient of variation $(\mathrm{CV})<15 \%$; inter-assay $\mathrm{CV}<18 \%$.

MR-proANP was measured in serum by TRACE technology (BRAHMS MR-proANP KRYPTOR assay, Kryptor Compact Plus; BRAHMS GmbH): LOD $2.1 \mathrm{pmol} \cdot \mathrm{L}^{-1}$; SCR 2.1 to $10000 \mathrm{pmol} \cdot \mathrm{L}^{-1}$; intra-assay CV $\leqslant 5 \%$; inter-assay $\mathrm{CV} \leqslant 6.5 \%$.

Serum N-terminal pro-B-type natriuretic peptide (NT-proBNP) concentrations were measured using an electrochemiluminescence immunoassay (NT-proBNP assay, Elecsys Analyser 2010; Roche Diagnostics, Mannheim, Germany): LOD $5 \mathrm{ng} \cdot \mathrm{L}^{-1}$; SCR 5 to $35000 \mathrm{ng} \cdot \mathrm{L}^{-1}$. The lowest concentration measurable with a CV of $20 \%$ is $50.0 \mathrm{ng} \cdot \mathrm{L}^{-1}$. At the cut-off value of $150 \mathrm{ng} \cdot \mathrm{L}^{-1}$ the $\mathrm{CV}$ is $<3 \%$.

\section{Statistical analysis}

All continuous variables are expressed as mean \pm standard deviation (SD) or as median and interquartile range (IQR), as appropriate. Categorical variables are reported as number and percentage. Parametric distribution was assessed using the Shapiro-Wilk test. Subcohorts at baseline, prior to PEA/BPA therapy, or at the follow-up were compared using the t-test for normally distributed parameters and the Mann-Whitney U-test for all other continuous variables. The Chi-squared test and Fisher-Yates test were used for categorical variables. Parameters that were obtained at baseline and at the 6-/12-MFU were subjected to paired sample testing. We used $t$-test for normally distributed parameters and the Wilcoxon signed-rank test for other continuous variables.

Correlations were analysed using bivariate Spearman correlation (Pearson's $\rho$ ). For further assessment of an association between biomarkers and haemodynamics, the cohort was divided into tertiles according to the degree of impairment of each haemodynamic parameter and biomarker levels were analysed in each subgroup.

Two predefined study outcomes were assessed:

1. Severe disease: In accordance with the recommendations in the guidelines for diagnosis and treatment of pulmonary hypertension [1], this outcome was defined as a right atrial pressure (RAP) $\geqslant 8 \mathrm{mmHg}$ in combination with a cardiac index $\leqslant 2.4 \mathrm{~L} \cdot \mathrm{min}^{-1} \cdot \mathrm{m}^{-2}$.

2. Optimal therapy response: This was defined as pulmonary haemodynamics below the thresholds of pulmonary hypertension (mean PAP $\leqslant 25 \mathrm{mmHg}$, PVR $\leqslant 3 \mathrm{WU}$ ) [1] and a normalised RAP $(\leqslant 6 \mathrm{mmHg}$ ) [26] or at least a distinct reduction in all of the three haemodynamic parameters (mean $\mathrm{PAP} \geqslant 25 \%, \mathrm{PVR} \geqslant 35 \%$ and $\mathrm{RAP} \geqslant 25 \%$ ) after PEA or BPA. The rational for this parameter selection was to address pulmonary haemodynamics (PVR, mean PAP) as well as a recovery of right heart failure (RAP), which is one major determinant of outcome in CTEPH.

The diagnostic performance of noninvasive biomarkers and certain other diagnostic findings to indicate study outcomes was analysed using receiver operating characteristics (ROC). Results are presented as AUC with corresponding 95\% confidence intervals. The optimal cut-off values with regard to study outcomes were calculated using Youden index quantification. AUCs were compared using DeLong test.

To assess the prognostic performance of optimal biomarker cut-off levels with regard to study outcomes, sensitivity, specificity, and negative (NPV) and positive (PPV) predictive values were calculated. Results are presented as odds ratios with corresponding $95 \%$ confidence intervals.

Statistics were performed with SPSS software (IBM Corp., Armonk, NY, USA), version 21.0. A two-tailed p-value $<0.05$ was considered to be statistically significant.

\section{Results}

\section{Patient characteristics, biomarker levels, treatment and effects of therapy}

The sociodemographic and clinical data of all 125 patients (51 women; mean age $( \pm \mathrm{SD}) 59 \pm 14$ years) enrolled in the study are presented in table 1. The indications for an interventional treatment were peripheral lesions in 65 (93\%) patients and persisting pulmonary artery obstructions after prior PEA in 5 (7\%) patients. The 70 patients who were allocated for an interventional treatment underwent a total of 413 BPA sessions (median 6 (5-7) per patient). Table 2 shows the effects of therapy on haemodynamics and 
Parameter

$\begin{array}{lc}\text { Subjects } \mathbf{n} & 125 \\ \text { Age years, } & 59 \pm 14 \\ \text { Female sex } & 51(40.8) \\ \text { Body mass index } \mathbf{~ k g} \cdot \mathbf{m}^{-\mathbf{2}} & 25.9 \pm 4.5 \\ \text { Chronic renal failure } & 26(20.8) \\ \quad \text { Estimated glomerular filtration rate } \mathrm{mL} \cdot \mathrm{min}^{-1} & 82.5 \pm 25.7 \\ \quad \text { Creatinine } \mu \mathrm{mol} \cdot \mathrm{L}^{-1} & 86 \pm 27 \\ \text { Coronary artery disease } & 20(16.0) \\ \text { History of cancer } & 18(14.4) \\ \text { Chronic obstructive pulmonary disease } & 8(6.4) \\ \text { History acute pulmonary embolism } & 110(88.0) \\ \text { History of isolated deep vein thrombosis } & 18(14.4) \\ \text { History of splenectomy } & 9(7.2) \\ \text { History of chronic inflammatory bowel disease } & 1(<1) \\ \text { Systemic inflammatory disease } & 2(1.6)\end{array}$

Values are presented as $\mathrm{n}(\%)$ or mean \pm SD, unless otherwise stated.

physical capacity. The beneficial effects on haemodynamics were more apparent 12 months after PEA compared with 6 months after BPA.

The measured serum biomarker levels of MR-proANP, copeptin and NT-proBNP at baseline are illustrated in table 3. No differences between PEA and BPA patients were observed: MR-proANP $(p=0.66)$, copeptin $(p=0.52)$, NT-proBNP $(p=0.63)$. MR-proANP levels correlated with PVR $(\rho=0.51 ; p<0.001)$, RAP $(\rho=0.51$; $p<0.001)$ and mean pulmonary arterial pressure (mPAP) $(\rho=0.44 ; p<0.001)$, whereas copeptin showed no relevant correlations with haemodynamics (table 4). Correlations with other diagnostic findings at baseline are also illustrated in table 4. The levels of MR-proANP and copeptin in subgroups divided according to the degree of haemodynamic impairment (divided into tertiles) are illustrated in figures la-d (MR-proANP) and figures le-h (copeptin).

TABLE 2 Comparison of diagnostic findings at baseline and 6-month (balloon pulmonary angioplasty (BPA)) or 12-month (pulmonary endarterectomy (PEA)) follow-up

\begin{tabular}{|c|c|c|c|c|c|c|c|}
\hline \multirow[t]{2}{*}{ Parameter } & \multicolumn{3}{|c|}{ Baseline } & \multicolumn{3}{|c|}{ 6-MFU/12-MFU } & \multirow[t]{2}{*}{ p-value (total) } \\
\hline & Total & PEA & BPA & Total & PEA & BPA & \\
\hline \multicolumn{8}{|l|}{ WHO-FC n } \\
\hline । & 0 & 0 & 0 & 69 & 30 & 39 & \\
\hline II & 14 & 14 & 0 & 45 & 22 & 23 & \\
\hline III & 78 & 31 & 47 & 9 & 3 & 6 & \\
\hline IV & 33 & 10 & 23 & 2 & 0 & 2 & \\
\hline RAP mmHg & $7(5-9)$ & $7(5-9)$ & $7(5-9)$ & $5(4-7)$ & $5(4-7)$ & $5(4-7)$ & $<0.001$ \\
\hline PCWP mmHg & $9(8-12)$ & $9(8-13)$ & $9(8-11)$ & $9(7-11)$ & $9(7-12) /$ & $9(8-11)$ & 0.175 \\
\hline Mean PAP mmHg & $42(36-49)$ & $44(36-50)$ & $40(36-49)$ & $25(20-33)$ & $20(17-24)$ & $30(25-35)$ & $<0.001$ \\
\hline Mean PAP reduction $\%$ & & & & 35 (21-53) & $53(36-63)$ & $10(6-16)$ & \\
\hline PVR Wood units & $6.8(5.3-9.6)$ & $7.1(5.2-11.9)$ & $6.8(5.2-8.6)$ & $3.4(2.4-4.9)$ & $2.5(1.8-3.5)$ & $3.9(3.0-5.3)$ & $<0.001$ \\
\hline PVR reduction \% & & & & $46(25-63)$ & $63(40-81)$ & $40(23-54)$ & \\
\hline $\mathrm{CI} \mathrm{L} \cdot \mathrm{min}^{-1} \cdot \mathrm{m}^{-2}$ & $2.4(2.1-2.8)$ & $2.3(1.9-2.6)$ & $2.6(2.1-3.0)$ & $2.6(2.3-2.9)$ & $2.4(2.3-2.8)$ & $2.7(2.4-3.0)$ & 0.009 \\
\hline 6MWD m & $405 \pm 99$ & $409 \pm 145$ & $410 \pm 101$ & $445 \pm 113$ & $438 \pm 129$ & $449 \pm 106$ & $<0.001$ \\
\hline LVEF \% & $55(55-60)$ & $55(55-60)$ & $55(55-60)$ & $55(55-60)$ & 55 (55-58) & $55(55-60)$ & 0.21 \\
\hline TAPSE mm & $19 \pm 5$ & $19 \pm 6$ & $19 \pm 5$ & $19 \pm 5$ & $17 \pm 4$ & $21 \pm 4$ & 0.90 \\
\hline
\end{tabular}

Data are presented as $\mathrm{n}$, mean \pm SD or median (interquartile range), unless otherwise stated. 6-/12-MFU: 6-/12-month follow-up; WHO: World Health Organization; FC: functional class; RAP: right atrial pressure; PCWP: pulmonary capillary wedge pressure; PAP: pulmonary arterial pressure; PVR: pulmonary vascular resistance; CI: cardiac index; 6MWD: 6-min walk distance; LVEF: left ventricular ejection fraction; TAPSE: tricuspid annular plane systolic excursion. 
TABLE 3 Comparison of biomarker findings at baseline and 6-month (balloon pulmonary angioplasty (BPA)) or 12-month (pulmonary endarterectomy (PEA)) follow-up

\begin{tabular}{|c|c|c|c|c|c|c|c|}
\hline \multirow[t]{2}{*}{ Parameter } & \multicolumn{3}{|c|}{ Baseline } & \multicolumn{3}{|c|}{ 6-MFU/12-MFU } & \multirow{2}{*}{$\begin{array}{l}\text { p-value } \\
\text { (total) }\end{array}$} \\
\hline & Total & PEA & BPA & Total & PEA & BPA & \\
\hline MR-proANP pmol-L'-1 & $156(91-246)$ & 170 (97-243) & $145(86-246)$ & 99 (58-145) & $98(57-160)$ & $101(59-142)$ & $<0.001$ \\
\hline Copeptin pmol. $\mathrm{L}^{-1}$ & $7.7(4.6-14.2)$ & $8.0(4.4-14.7)$ & $7.1(4.6-13.5)$ & $6.3(3.7-12.6)$ & $6.2(3.8-13.7)$ & $6.4(3.7-12.2)$ & 0.009 \\
\hline NT-proBNP $n g \cdot \mathrm{L}^{-1}$ & 845 (178-1875) & $1094(136-2163)$ & $744(195-1564)$ & 142 (72-335) & $192(98-408)$ & $121(67-243)$ & $<0.001$ \\
\hline Creatinine $\mu \mathrm{mol} \cdot \mathrm{L}^{-1}$ & $86 \pm 28$ & $88 \pm 31$ & $84 \pm 0.30$ & $80 \pm 26$ & $81 \pm 31$ & $78 \pm 21$ & $<0.001$ \\
\hline $\begin{array}{l}\text { eGFR } \mathrm{mL} \cdot \mathrm{min}^{-1} \cdot \mathrm{m}^{-2} \\
\text { eGFR } \leqslant 60 \mathrm{~mL} \cdot \mathrm{min}^{-1} \cdot \mathrm{m}^{-2}\end{array}$ & $82.5 \pm 25.7$ & $\begin{array}{l}81.1 \pm 24.7 \\
21(16.8)\end{array}$ & $83.6 \pm 26.6$ & $91.7 \pm 37.7$ & $\begin{array}{l}92.0 \pm 27.1 \\
12(9.6)\end{array}$ & $91.5 \pm 44.5$ & $<0.001$ \\
\hline
\end{tabular}

Data are presented as median (interquartile range), mean \pm SD or $n(\%)$, unless otherwise stated. 6-/12-MFU: 6-/12-month follow-up; MR-proANP: mid-regional pro-atrial natriuretic peptide; NT-proBNP: N-terminal pro-B-type natriuretic peptide; eGFR: estimated glomerular filtration rate.

Identification of patients with more severe disease (primary outcome)

Haemodynamically compromised patients with severe disease $(\mathrm{n}=17)$ had higher baseline levels of MR-proANP (320 (246-527) pmol. $\mathrm{L}^{-1}$ versus $\left.133(82-215) \mathrm{pmol} \cdot \mathrm{L}^{-1} ; \mathrm{p}=0.001\right)$, copeptin $([12.7(7.3-20.6)$ pmol. $\mathrm{L}^{-1}$ versus $\left.6.8(4.4-12.8) \mathrm{pmol} \cdot \mathrm{L}^{-1} ; \mathrm{p}=0.015\right)$ and NT-proBNP $\left(2986(1474-5255) \mathrm{ng} \cdot \mathrm{L}^{-1}\right.$ versus 544 (110-1393) $\left.\mathrm{ng} \cdot \mathrm{L}^{-1} ; \mathrm{p}=0.001\right)$. The strongest association between biomarker concentration and severe disease was found for MR-proANP levels $\geqslant 227 \mathrm{pmol} \cdot \mathrm{L}^{-1}$ (AUC 0.91; OR 56 (95\% CI 6.9-454.3)) and

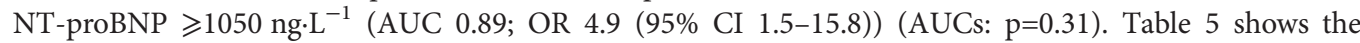
diagnostic performance of other diagnostic findings to identify patients with severe disease.

\section{Biomarker dynamics after PEA/BPA}

Table 3 illustrates the comparison of serum biomarker levels at baseline and at follow-up. In the PEA cohort, MR-proANP $(\mathrm{p}=0.001)$, copeptin $(\mathrm{p}=0.017)$ and NT-proBNP $(\mathrm{p}<0.001)$ levels decreased from baseline to 12-MFU. In the BPA cohort, MR-proANP $(\mathrm{p}<0.001)$ and NT-proBNP $(\mathrm{p}<0.001)$ decreased significantly from baseline to $6-\mathrm{MFU}$, but copeptin did not change $(\mathrm{p}=0.18)$. The analysis of biomarker level dynamics during the staged BPA procedures showed a continuous decrease in MR-proANP levels at all pre-specified time points following the first BPA, with the lowest value being measured at the 6-MFU (figure 2), but no dynamics of copeptin levels (supplementary figure).

\section{Identification of patients with optimal therapy response (secondary outcome)}

The strongest association between biomarker concentration and therapy response was observed for MR-proANP levels $\leqslant 123 \mathrm{pmol} \cdot \mathrm{L}^{-1}$ (AUC 0.70; OR 5.2, 95\% CI 2.0-13.5) and NT-proBNP $\leqslant 369 \mathrm{ng} \cdot \mathrm{L}^{-1}$

TABLE 4 Bivariate Spearman correlation of biomarker levels and other diagnostic findings at baseline

\begin{tabular}{|c|c|c|}
\hline Parameter & MR-proANP pmol. $\mathrm{L}^{-1}$ & Copeptin pmol. $\mathrm{L}^{-1}$ \\
\hline Age years & $\rho=0.20 ; p=0.03$ & $\rho=0.28 ; p=0.002$ \\
\hline Body mass index $\mathrm{kg} \cdot \mathrm{m}^{-2}$ & $\rho=-0.29 ; p=0.001$ & $\rho=-0.06 ; p=0.53$ \\
\hline GFR $\mathrm{mL} \cdot \mathrm{min}^{-1}$ & $\rho=-0.31 ; p<0.001$ & $\rho=-0.27 ; p=0.003$ \\
\hline Serum creatinine $\mu \mathrm{mol} \cdot \mathrm{L}^{-1}$ & $\rho=0.31 ; p<0.001$ & $\rho=0.35 ; p<0.001$ \\
\hline mPAP mmHg & $\rho=0.44 ; p<0.001$ & $\rho=0.07 ; p=0.47$ \\
\hline PVR WU & $\rho=0.51 ; p<0.001$ & $\rho=0.07 ; p=0.46$ \\
\hline RAP mmHg & $\rho=0.51 ; p<0.001$ & $\rho=0.26 ; p=0.008$ \\
\hline $\mathrm{Cl} \cdot \mathrm{min}^{-1} \cdot \mathrm{m}^{-2}$ & $\rho=-0.36 ; p<0.001$ & $\rho=-0.13 ; p=0.15$ \\
\hline 6MWD m & $\rho=-0.26 ; p=0.03$ & $\rho=-0.27 ; p=0.02$ \\
\hline LVEF \% & $\rho=-0.30 ; p=0.002$ & $\rho=-0.03 ; p=0.74$ \\
\hline TAPSE $\mathrm{mm}$ & $\rho=-0.28 ; p=0.004$ & $\rho=-0.20 ; p=0.04$ \\
\hline
\end{tabular}

MR-proANP: mid-regional pro-atrial natriuretic peptide; GFR: glomerular filtration rate; mPAP: mean pulmonary arterial pressure; PVR: pulmonary vascular resistance; RAP: right atrial pressure; $\mathrm{Cl}$ : cardiac index; 6MWD: 6-min walk distance; LVEF: left ventricular ejection fraction; TAPSE: tricuspid annular plane systolic excursion. 

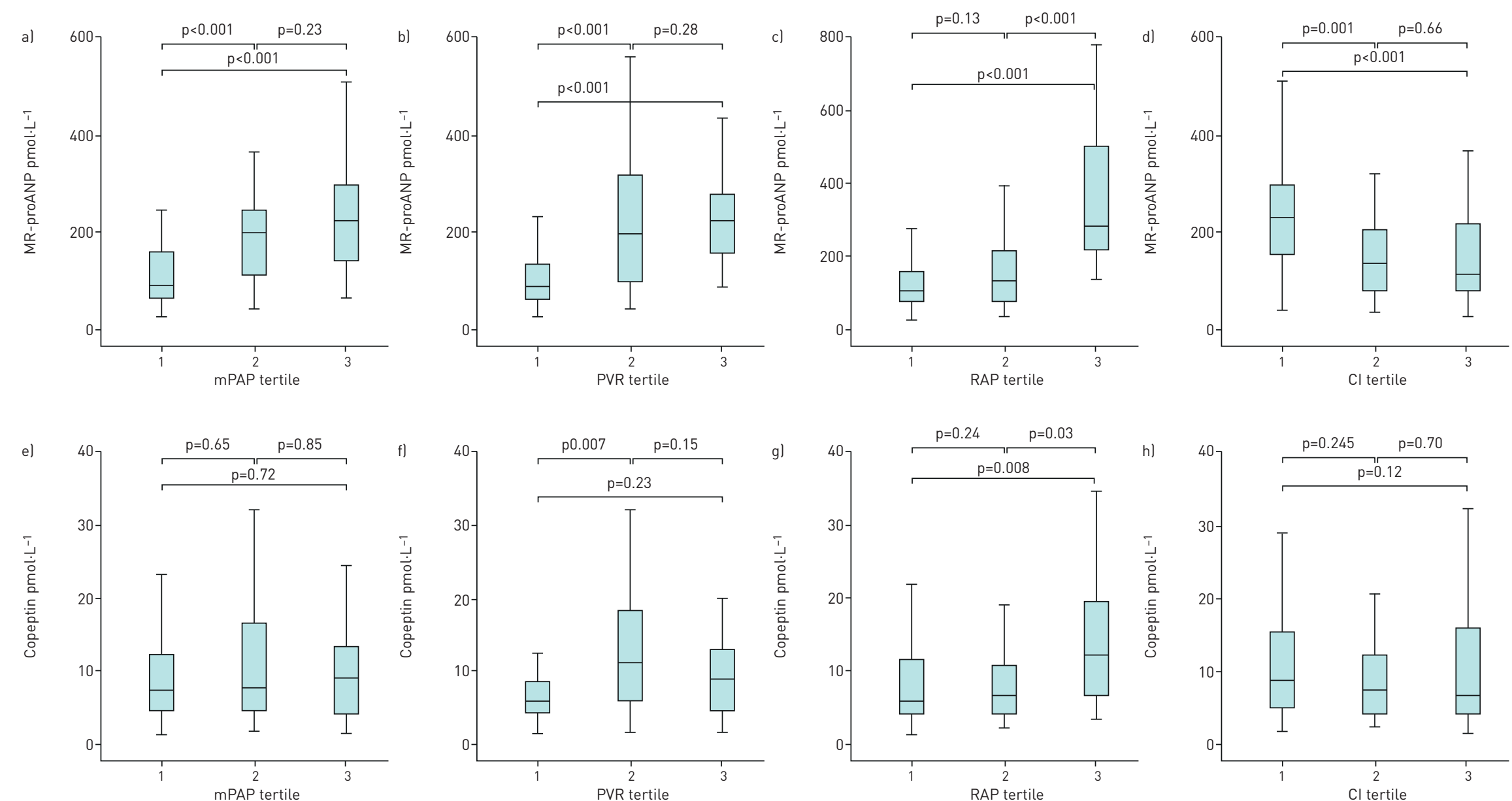

FIGURE 1 Biomarker levels (a-d: mid-regional pro-atrial natriuretic peptide (MR-proANP); e-h: copeptin) as a function of haemodynamic parameter. The cohort of $\mathrm{n}=125$ chronic thromboembolic pulmonary hypertension patients was divided into tertiles for each haemodynamic parameter according to the following cut-off values: a, e) mean pulmonary arterial pressure (mPAP; $\leqslant 38 / \leqslant 47 \mathrm{mmHg}$ ); b, f) pulmonary vascular resistance (PVR; $\leqslant 5.7 / \leqslant 8.3 \mathrm{WU}$ ); c, g) right atrial pressure (RAP; $\leqslant 5 / \leqslant 8 \mathrm{mmHg}$; and $d$, h) cardiac index (Cl; $\leqslant 2.1 / \leqslant 2.7 \mathrm{~L} \cdot \mathrm{min}^{-1} \cdot \mathrm{m}^{-2}$ ). 
TABLE 5 Prognostic performance of different parameters for the identification of patients with severe disease (primary outcome) and optimal therapy response (secondary outcome)

\section{Cut-off value \\ AUC $(95 \% \mathrm{Cl})$ \\ Sensitivity \\ $(\%, 95 \% \mathrm{Cl})$}

Specificity

$(\%, 95 \% \mathrm{CI})$
NPV

$(\%, 95 \% \mathrm{Cl})$
PPV $(\%, 95 \% \mathrm{CI})$
OR

(95\% CI)

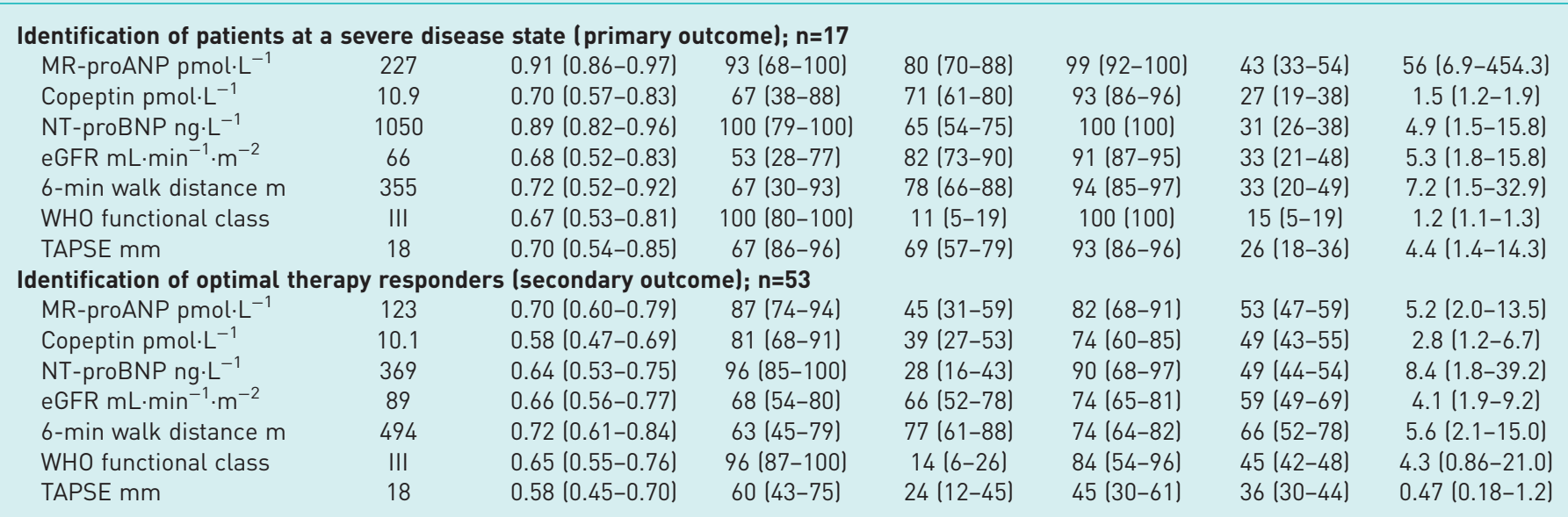

AUC: area under the curve; $\mathrm{Cl}$ : confidence interval; NPV: negative predictive values; PPV: positive predictive values; OR: odds ratio; MR-proANP: mid-regional pro-atrial natriuretic peptide; NT-proBNP: N-terminal pro-B-type natriuretic peptide; eGFR: estimated glomerular filtration rate; WHO: World Health Organization; TAPSE: tricuspid annular plan systolic excursion.

(AUC 0.64; OR 8.4, 95\% CI 1.8-39.2) (AUC: $\mathrm{p}=0.83$ ). Table 5 illustrates the diagnostic performance of biomarker levels measured at 6-/12-MFU and other diagnostic findings to identify patients with optimal therapy response after PEA or BPA $(n=53)$. In total, $34(27.2 \%)$ of the patients showed an mPAP $\leqslant 20 \mathrm{mmHg}$ at the follow-up.

\section{Discussion}

In the course of CTEPH disease progression, cardiac compensatory adaption mechanisms gradually fail, which leads to right heart failure [2,4]. This eventually affects the systemic circulation, which leads to a deteriorating clinical status and strongly correlates with adverse outcome [2-5]. Therefore, noninvasive measurement of biomarkers that mirror haemodynamic conditions and right heart stress is a valuable adjunct for individual assessment of disease severity, risk stratification and therapy monitoring $[1,7-9,27]$.

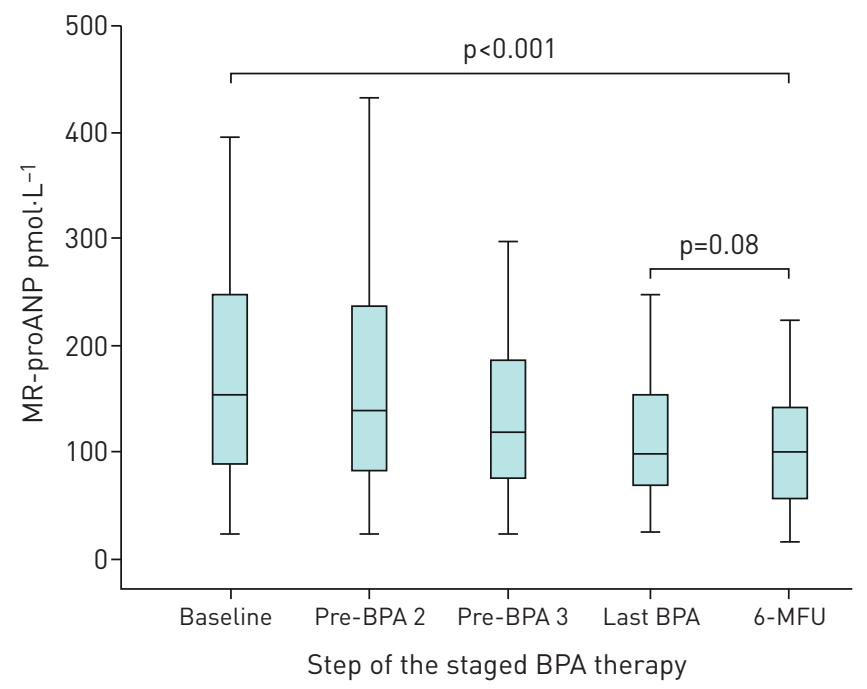

FIGURE 2 Dynamics of mid-regional pro-atrial natriuretic peptide (MR-proANP) levels during staged balloon pulmonary angioplasty (BPA) procedures. Biomarker measure-ment was carried out at the specified stage in BPA therapy of 55 chronic thromboembolic pulmonary hypertension patients. 6-MFU: 6-month follow-up. 


\section{Key findings}

We determined that baseline MR-proANP levels are associated with disease severity defined by haemodynamic parameters in CTEPH and decrease after PEA/BPA. Copeptin levels are lower in CTEPH than in acute cardiovascular disease and do not substantially change after therapy. MR-proANP was associated with an AUC of 0.91 in ROC analysis for the identification of patients with impaired haemodynamics and thus severe disease (primary outcome) at baseline. MR-proANP identified patients with optimal therapy response (secondary outcome) with an AUC of 0.70 at the 6-MFU after BPA and at the 12-MFU after PEA.

\section{MR-proANP and copeptin in patients with CTEPH}

Natriuretic peptides are released from the myocardium in response to an increased wall tension due to volume or pressure overload $[11,15]$. Circulating levels of NT-proBNP strongly correlate with haemodynamic disease severity in pulmonary hypertension and CTEPH $[1,8,9,15,16,23]$. However, it should be noted that subtypes of natriuretic peptides show different expression characteristics [11]. In contrast to NT-proBNP, ANP mainly derives from the atria [11] and thus predominantly reflects right atrial stress. Right atrial dilatation, a consequence of chronic right ventricular dysfunction in pulmonary hypertension, is usually detected by imaging and has been shown to be a strong predictor of outcome [28]. Measurement of ANP/MR-proANP levels provides a simple and noninvasive way of obtaining this information. An MR-proANP level of $120 \mathrm{pmol} \cdot \mathrm{L}^{-1}$ was shown to rule out acute heart failure with a sensitivity $>90 \%$ [29]. In acute pulmonary embolism [17] and different forms of pulmonary hypertension $[15,16,23]$, the reported median MR-proANP level ranges from 105 to $201 \mathrm{pmol} \cdot \mathrm{L}^{-1}$ and was $130 \mathrm{pmol} \cdot \mathrm{L}^{-1}$ in a small subcohort of seven CTEPH patients [15]. The median MR-proANP level of 156 (91-246) $\mathrm{pmol} \cdot \mathrm{L}^{-1}$ in our cohort is comparable to these data and reflects chronic cardiac stress in the majority of CTEPH patients. The hypothesis that MR-proANP can be used as a noninvasive surrogate of atrial stress is supported by the correlation of MR-proANP levels and RAP in our cohort.

Besides expression characteristics, the independence of MR-proANP levels from obesity and anaemia might also be a useful feature in selected patients [30].

Vasopressin and copeptin are released from the hypothalamus in response to hypotension, systemic stress and changes in plasma osmolality [12]. Vasopressin acts as a fast regulator of fluid homeostasis through the promotion of renal salt-free fluid reabsorption and peripheral vasoconstriction [12]. Whereas cardiac wall stress $[8,10]$ and subacute myocardial ischaemia $[7,10]$ are reflected by elevated natriuretic peptides and cardiac troponins in CTEPH, vasopressin or its derivative copeptin might serve as a surrogate for the impairment of systemic circulation as a result of progressive right heart failure. In acute pulmonary embolism, median copeptin levels ranging between minimum values of 10 and $14 \mathrm{pmol} \cdot \mathrm{L}^{-1}$ up to values of $705 \mathrm{pmol} \cdot \mathrm{L}^{-1}$ were observed $[19,20]$. In studies including mixed cohorts of patients with pulmonary hypertension and only a very small number of CTEPH patients, the median copeptin level ranged from 8 to $20 \mathrm{pmol} \cdot \mathrm{L}^{-1}$, which is comparable to our observations $[16,22,23]$. In acute pulmonary embolism a copeptin level of $24 \mathrm{pmol} \cdot \mathrm{L}^{-1}$ was identified as the optimal cut-off value to predict adverse outcome [19], and a level of $10 \mathrm{pmol} \cdot \mathrm{L}^{-1}$ is used to rule out acute myocardial infarction [31]. In the present study, median copeptin levels at baseline $\left(8 \mathrm{pmol} \cdot \mathrm{L}^{-1}\right)$ and follow-up $\left(6 \mathrm{pmol} \cdot \mathrm{L}^{-1}\right)$ were low. This might be explained by the function of vasopressin as an acute, fast-acting regulator of haemodynamic imbalance and the fact that the majority of CTEPH patients were not in an acute, life-threatening situation with acute haemodynamic impairment at the time of admission (and study enrolment) for elective BPA or PEA.

If noninvasive biomarker measurement is used as a diagnostic tool, potential confounders need to be considered. Chronic renal failure frequently occurs as one negative side effect of pulmonary hypertension and right heart failure $[32,33]$. In this context, the decrease of renal clearance and the cardiac stress impacts biomarker levels [34]. This diagnostic limitation is moderate in patients with preserved renal function (estimated glomerular filtration rate $(\mathrm{eGFR})>60 \mathrm{~mL} \cdot \mathrm{min}^{-1} \cdot \mathrm{m}^{-2}$ ) whereas adapted cut-off values have to be used in patients with chronic renal failure [34]. In our cohort, the majority of patients had preserved renal function or slightly reduced eGFR at baseline. PEA and BPA lead to an improvement of renal function, which lowered the number of patients with an eGFR $\leqslant 60 \mathrm{~mL} \cdot \mathrm{min}^{-1} \cdot \mathrm{m}^{-2}$ from $21(16.8 \%)$ at baseline to $12(9.6 \%)$ at follow-up. Our data are not representative to demonstrate an impact of renal function on MR-proANP and copeptin levels. Both biomarkers correlated moderately with serum creatinine and eGFR at baseline in our cohort. Thus, the individual renal function should be considered when serum levels of both biomarkers are assessed.

\section{Biomarker-based identification of patients with severe disease}

Published data demonstrate the superiority of MR-proANP compared with established biomarkers for predicting an adverse outcome in chronic heart failure [13], pulmonary embolism [17] and pulmonary 
hypertension [16]. In heart failure, the additive measurement of MR-proANP improved disease detection and risk stratification compared to a sole measurement of brain natriuretic peptides $[35,36]$. In a cohort of 710 patients who were admitted due to dyspnoea, no other natriuretic peptide performed better than MR-proANP as a predictor of 5-year survival.

In our study, CTEPH patients with higher mPAP, PVR, RAP and lower cardiac index had higher levels of MR-proANP, which conforms with the data from a mixed pulmonary hypertension cohort of KAISER et al. [15]. Consistent with this, the 17 patients of our cohort with severe disease (primary outcome) had higher MR-pro-ANP levels than the rest of the cohort. Patients with a calculated optimal MR-proANP cut-off value $\geqslant 227 \mathrm{pmol} \cdot \mathrm{L}^{-1}$ had a 56 -fold increased risk of impaired haemodynamics and thus severe disease (95\% CI 6.9-454.3). In this context, MR-proANP performance was at least comparable to NT-proBNP and superior to that of copeptin and other diagnostic findings, including 6-min walking distance, World Health Organization functional class (WHO-FC) and tricuspid annular plane systolic excursion (TAPSE) (table 5). MR-proANP measurement addresses not only right heart stress in general but also right atrial stress and the associated retrograde right heart failure, which might be the strength of this parameter.

Certain studies have revealed an association of copeptin levels and outcome in pulmonary hypertension; however, this was predominantly with an inferior predictive value compared with natriuretic peptides [16, 23]. While copeptin levels correlated with functional capacity and clinical symptoms in cohorts of patients with different types of pulmonary hypertension $[16,22,23]$, the studies report inconsistent data concerning the correlation with pulmonary and systemic haemodynamics $[16,22]$. In our cohort, there was no relevant correlation of copeptin levels with haemodynamic parameters. The only association we observed was that patients with the highest RAP tertile had distinctly elevated copeptin levels at baseline. Accordingly, RAP was the main driver for the observed higher copeptin levels in patients with severe disease compared with the rest of the cohort. Elevation of copeptin above the calculated optimal cut-off level of $10.9 \mathrm{pmol} \cdot \mathrm{L}^{-1}$ was associated with an OR of only 1.5 (95\% CI 1.2-1.9) to identify patients with severe disease, which is inferior compared with MR-proANP, NT-proBNP and other diagnostic parameters.

\section{Identification of patients with optimal therapy response}

Data on MR-proANP levels after established therapy for pulmonary hypertension (secondary outcome), particularly after surgical or interventional CTEPH treatment, are not available. In the current study, PEA and BPA led to a decrease in MPAP and PVR that was comparable to previously published data from our group and other cohorts $[8,25,37]$. The consequently reduced right heart stress was mirrored by a decrease in MR-proANP levels in the current study, irrespective of the therapeutic approach. The time course of MR-proANP levels during BPA therapy with a stepwise decrease after each session and an ongoing decrease at the follow-up illustrates progressive recovery of retrograde right heart failure. An MR-proANP concentration of $123 \mathrm{pmol} \cdot \mathrm{L}^{-1}$ at follow-up was identified as the best threshold to identify patients with an optimal therapy response.

Less is known about the impact of pulmonary hypertension therapies on copeptin levels. A study by NiCKEL et al. [22] is the only one that reported decreasing levels of copeptin in patients with pulmonary artery hypertension who received targeted medication. In our study, a slight decrease in copeptin levels (from 8 to $6 \mathrm{pmol} \cdot \mathrm{L}^{-1}$ ) was observed after PEA, but not after BPA. Copeptin levels above the calculated optimal cut-off value of $10.1 \mathrm{pmol} \cdot \mathrm{L}^{-1}$ predicted an optimal haemodynamic therapy response; however, the overall prognostic performance was poor, as indicated by an AUC of only 0.58. As discussed above, this further indicates that vasopressin/copeptin is a better marker of acute haemodynamic compromising diseases rather than of chronic processes.

\section{Study limitations}

First, the absolute number of patients included in this study was relatively small with heterogeneous treatment groups. Second, the follow-up assessment after PEA and BPA is performed in different intervals after treatment, which might limit the comparability between the treatment groups. Third, follow-up in this study is limited to 12 months after PEA and 6 months after BPA. However, the results of the biomarker analysis are consistent between CTEPH patients treated with PEA and those treated with BPA.

\section{Conclusion}

Serum levels of MR-proANP are associated with haemodynamic disease severity in CTEPH patients, providing evidence in favour of using this biomarker for individual risk stratification and assessment of therapy response. Further, the continuous decrease in MR-proANP levels after each BPA session parallel the immediate and beneficial short-term effects of this therapy. However, the clinical applicability is limited at the current state. Further studies are needed to confirm the association of MR-proANP levels to 
progressive right heart failure, and long-term data are required to assess the performance as a predictor of outcome.

Copeptin showed a weak diagnostic performance in CTEPH and thus appears to be a marker of acute rather than chronic haemodynamic impairment.

Acknowledgements: We thank Elizabeth Martinson from the Kerckhoff Herzforschungsinstitut Editorial Office (Giessen, Germany) for her editorial assistance.

Conflict of interest: S.D. Kriechbaum has nothing to disclose. L. Scherwitz has nothing to disclose. C.B. Wiedenroth reports personal fees from Actelion, Bayer, MSD, Pfizer and BTG outside the submitted work. F. Rudolph has nothing to disclose. S. Wolter has nothing to disclose. M. Haas reports personal fees from Daiichi-Sankyo and Pfizer outside the submitted work. U. Fischer-Rasokat has nothing to disclose. A. Rolf reports personal fees from Astra Zeneca, Boehringer Ingelheim and Pfizer-Bristol-Myers Squibb outside the submitted work;. C.W. Hamm reports personal fees from BRAHMS/Thermo Fisher outside the submitted work. E. Mayer reports grants from Deutsche Forschungsgesellschaft during the conduct of the study; and personal fees from Actelion, Bayer, MSD and Pfizer outside the submitted work. S. Guth reports personal fees from Actelion, Bayer, GSK, MSD and Pfizer outside the submitted work. T. Keller reports personal fees from Abbott and Brahms outside the submitted work. S.V. Konstantinides reports grants from German Federal Ministry of Education and Research (BMBF 01EO1503) during the conduct of the study; and grants and personal fees from Bayer, personal fees from Pfizer Bristol Myers Suqibb, grants and personal fees from Boehringer Ingelheim, personal fees from Daiichi Sankyo and MSD, and grants from Actelion, outside the submitted work;. M. Lankeit reports grants from German Federal Ministry of Education and Research (BMBF 01EO1503) during the conduct of the study; and grants and personal fees from Bayer, personal fees from Pfizer Bristol Myers Squibb, personal fees from Daiichi Sankyo and MSD, grants and personal fees from Thermo Fisher Scientific, and personal fees from Actelion, outside the submitted work. C. Liebetrau reports personal fees from Bayer, Pfizer-Bristol-Myers Squibb, GSK, Abott, AstraZeneca, Berlin Chemie, Daiichi-Sankyo and Boehringer Ingelheim outside the submitted work.

Support statement: This research project is based on a cohort that is part of the Kerckhoff Biomarker Registry (Bioreg) that is financially supported by the Kerckhoff Heart Research Institute (KHFI) and the German Center for Cardiovascular Research (DZHK). We are also grateful to the William G. Kerckhoff Foundation (Bad Nauheim, Germany) for research funding. The current evaluation is supported by the grant of the Kerckhoff Foundation and is associated with the collaborative research centre 1213 (SFB 1213). The sponsors had no influence on the study design, statistical analyses or drafting of the manuscript. The work of M. Lankeit and S.V. Konstantinides was supported by the German Federal Ministry of Education and Research (BMBF 01EO1503). The authors are responsible for the contents of this publication. BRAHMS GmbH, part of Thermo Fisher Scientific (Hennigsdorf/Berlin, Germany) provided the assays for MR-proANP and copeptin measurements. The sponsor was neither involved in biomarker measurements, statistical analyses, writing of the manuscript nor had any influence on the scientific contents of this publication. Funding information for this article has been deposited with the Crossref Funder Registry.

\section{References}

1 Galiè N, Humbert M, Vachiery J-L, et al. 2015 ESC/ERS Guidelines for the diagnosis and treatment of pulmonary hypertension. The Joint Task Force for the Diagnosis and Treatment of Pulmonary Hypertension of the European Society of Cardiology (ESC) and the European Respiratory Society (ERS): Endorse. Eur Heart J 2016; 37: 67-119.

2 Matthews DT, Hemnes AR. Current concepts in the pathogenesis of chronic thromboembolic pulmonary hypertension. Pulm Circ 2016; 6: 145-154.

3 Mulchrone A, Kellihan HB, Forouzan $\mathrm{O}$, et al. A large animal model of right ventricular failure due to chronic thromboembolic pulmonary hypertension: a focus on function. Front Cardiovasc Med 2018; 5: 189.

4 Simonneau G, Torbicki A, Dorfmuller P, et al. The pathophysiology of chronic thromboembolic pulmonary hypertension. Eur Respir Rev 2017; 26: 160112.

5 Delcroix M, Lang I, Pepke-Zaba J, et al. Long-term outcome of patients with chronic thromboembolic pulmonary hypertension (CTEPH): results from an international prospective registry. Circulation 2016; 133: 859-871.

6 Wilkens H, Konstantinides S, Lang IM, et al. Chronic thromboembolic pulmonary hypertension (CTEPH) updated recommendations from the Cologne Consensus Conference 2018. Int J Cardiol 2018; 272s: 69-78.

7 Kriechbaum SD, Wiedenroth CB, Keller T, et al. Dynamics of high-sensitivity cardiac troponin T during therapy with balloon pulmonary angioplasty for chronic thromboembolic pulmonary hypertension. PLoS One 2018; 13: e0204683.

8 Kriechbaum SD, Wiedenroth CB, Wolter JS, et al. N-terminal pro-B-type natriuretic peptide for monitoring after balloon pulmonary angioplasty for chronic thromboembolic pulmonary hypertension. J Heart Lung Transpl 2018; 37: 639-646.

9 Andreassen AK, Wergeland R, Simonsen S, et al. N-terminal pro-B-type natriuretic peptide as an indicator of disease severity in a heterogeneous group of patients with chronic precapillary pulmonary hypertension. Am J Cardiol 2006; 98: 525-529.

10 Andreassen AK, Ragnarsson A, Gude E, et al. Balloon pulmonary angioplasty in patients with inoperable chronic thromboembolic pulmonary hypertension. Heart 2013; 99: 1415-1420.

11 Nakagawa Y, Nishikimi T, Kuwahara K. Atrial and brain natriuretic peptides: hormones secreted from the heart. Peptides 2019; 111: 18-25.

12 Bolignano D, Cabassi A, Fiaccadori E, et al. Copeptin (CTproAVP), a new tool for understanding the role of vasopressin in pathophysiology. Clin Chem Lab Med 2014; 52: 1447-1456.

13 von Haehling S, Jankowska EA, Morgenthaler NG, et al. Comparison of midregional pro-atrial natriuretic peptide with $\mathrm{N}$-terminal pro-B-type natriuretic peptide in predicting survival in patients with chronic heart failure. J Am Coll Cardiol 2007; 50: 1973-1980.

14 Kiely DG, Kennedy NS, Pirzada O, et al. Elevated levels of natriuretic peptides in patients with pulmonary thromboembolism. Respir Med 2005; 99: 1268-1291. 
15 Kaiser R, Grotemeyer K, Lepper P, et al. Associations of circulating natriuretic peptides with haemodynamics in precapillary pulmonary hypertension. Respir Med 2015; 109: 1213-1223.

16 Kolditz M, Seyfarth HJ, Wilkens H, et al. MR-proADM predicts exercise capacity and survival superior to other biomarkers in PH. Lung 2015; 193: 901-910.

17 Öner Ö, Deveci F, Telo S, et al. MR-proADM and MR-proANP levels in patients with acute pulmonary embolism. J Med Biochem 2019; 38: 1-8.

18 Mockel M, Searle J, Hamm C, et al. Early discharge using single cardiac troponin and copeptin testing in patients with suspected acute coronary syndrome (ACS): a randomized, controlled clinical process study. Eur Heart J 2015 36: 369-376.

19 Hellenkamp K, Pruszczyk P, Jimenez D, et al. Prognostic impact of copeptin in pulmonary embolism: a multicentre validation study. Eur Respir J 2018; 51: 1702037.

20 Hellenkamp K, Schwung J, Rossmann H, et al. Risk stratification of normotensive pulmonary embolism: prognostic impact of copeptin. Eur Respir J 2015; 46: 1701-1710.

21 Hori Y, Uchide T, Saitoh R, et al. Diagnostic utility of NT-proBNP and ANP in a canine model of chronic embolic pulmonary hypertension. Vet J 2012; 194: 215-221.

22 Nickel NP, Lichtinghagen R, Golpon $\mathrm{H}$, et al. Circulating levels of copeptin predict outcome in patients with pulmonary arterial hypertension. Respir Res 2013; 14: 130.

23 Silva Marques J, Martins SR, Calisto C, et al. An exploratory panel of biomarkers for risk prediction in pulmonary hypertension: emerging role of CT-proET-1. J Heart Lung Transpl 2013; 32: 1214-1221.

24 Wiedenroth CB, Ghofrani HA, Adameit MSD, et al. Sequential treatment with riociguat and balloon pulmonary angioplasty for patients with inoperable chronic thromboembolic pulmonary hypertension. Pulm Circ 2018; 8: 2045894018783996

25 Guth S, Wiedenroth $\mathrm{CB}$, Kramm $\mathrm{T}$, et al. Pulmonary endarterectomy for the treatment of chronic thromboembolic pulmonary hypertension. Expert Rev Respir Med 2016; 10: 673-684.

26 Ragosta M, Kennedy JLW. Normal waveforms, artifacts, and pitfalls. In: Ragosta M, ed. Textbookof Clinical Hemodynamics. 2nd Edn. Philadelphia, Elsevier, 2018; pp. 17-55.

27 Kriechbaum SD, Wiedenroth CB, Hesse ML, et al. Development of renal function during staged balloon pulmonary angioplasty for inoperable chronic thromboembolic pulmonary hypertension. Scand J Clin Lab Invest 2019; 79: 268-275.

28 Raymond RJ, Hinderliter AL, Willis PW IV, et al. Echocardiographic predictors of adverse outcomes in primary pulmonary hypertension. J Am Coll Cardiol 2002; 39: 1214-1219.

29 Daniels LB, Clopton P, Potocki M, et al. Influence of age, race, sex, and body mass index on interpretation of midregional pro atrial natriuretic peptide for the diagnosis of acute heart failure: results from the $\mathrm{BACH}$ multinational study. Eur J Heart Fail 2012; 14: 22-31.

30 Sinning C, Ojeda F, Wild PS, et al. Midregional proadrenomedullin and growth differentiation factor-15 are not influenced by obesity in heart failure patients. Clin Res Cardiol 2017; 106: 401-410.

31 Keller T, Tzikas S, Zeller T, et al. Copeptin improves early diagnosis of acute myocardial infarction. J Am Coll Cardiol 2010; 55: 2096-2106.

32 Bitker L, Sens F, Payet C, et al. Presence of kidney disease as an outcome predictor in patients with pulmonary arterial hypertension. Am J Nephrol 2018; 47: 134-143.

33 Nickel NP, O'Leary JM, Brittain EL, et al. Kidney dysfunction in patients with pulmonary arterial hypertension. Pulm Circ 2017; 7: 38-54.

34 Han X, Zhang S, Chen Z, et al. Cardiac biomarkers of heart failure in chronic kidney disease. Clin Chim Acta 2020; 510: 298-310.

35 Seronde MF, Gayat E, Logeart D, et al. Comparison of the diagnostic and prognostic values of B-type and atrial-type natriuretic peptides in acute heart failure. Int J Cardiol 2013; 168: 3404-3411.

36 Masson S, Latini R, Carbonieri E, et al. The predictive value of stable precursor fragments of vasoactive peptides in patients with chronic heart failure: data from the GISSI-heart failure (GISSI-HF) trial. Eur J Heart Fail 2010 12: $338-347$.

37 Wiedenroth $\mathrm{CB}$, Olsson KM, Guth S, et al. Balloon pulmonary angioplasty for inoperable patients with chronic thromboembolic disease. Pulm Circ 2018; 8: 2045893217753122. 